\title{
Proceeding
}

Supplementary Issue: Winter Conferences of Sports Science. Costa Blanca Sports Science Events, 22-23 March 2021. Alicante, Spain.

\section{Effects of functional and traditional training on body composition and chronic low back pain treatment: Case study}

\author{
GENTI PANO ${ }^{1} \triangle$, ANDIS BOGDANI ${ }^{2}$
}

${ }^{1}$ Department of Kinesiology, Faculty of Rehabilitation Sciences, Sports University of Tirana, Tirana, Albania ${ }^{2}$ Department of Movement and Health, Faculty of Physical Activity and Recreation, Sports University of Tirana, Tirana, Albania

\begin{abstract}
Low back pain is a common musculoskeletal symptom that may be either acute or chronic. It may be caused by a variety of diseases and disorders that affect the lumbar spine. Exercise can improve back extension strength, mobility, endurance, and functional disability. (Manniche C et al., 1988; Manniche C et al., 1991). Participant in this case study was a 33 old female subject that presented complaining for severe chronic low back pain in the past two months. Anthropometric, body composition (BIA) spinal X-ray and MRI were conducted before and after intervention. Results show a statistically significant improvement in: Total body weight from $109.1 \mathrm{~kg}$ to $90.8 \mathrm{~kg}$, BMl from 36 to 29.47 , Total BF (\%) from 43.7 to $29(p<.05)$. The exercise program helped her to improve her daily life and be more productive in her work task. Exercise functional and traditional training program had a positive effect on body composition and in decreasing chronic low back pain. It is important that these exercise training programs to be prescribed from appropriate specialist and should be personalized for the specific individual needs in order to achieve the best results.
\end{abstract}

Keywords: Functional training; Traditional training; Body composition; Low back pain.

Cite this article as:

Pano, G., \& Bogdani, A. (2021). Effects of functional and traditional training on body composition and chronic low back pain treatment: Case study. Journal of Human Sport and Exercise, 16(3proc), S1395-S1401. https://doi.org/10.14198/jhse.2021.16.Proc3.55

Corresponding author. Department of Kinesiology, Faculty of Rehabilitation Sciences, Sports University of Tirana, Tirana, Albania.

E-mail: g.pano@ust.edu.al

Abstract submitted to: Winter Conferences of Sports Science. Costa Blanca Sports Science Events, 22-23 March 2021. Alicante, Spain.

JOURNAL OF HUMAN SPORT \& EXERCISE ISSN 1988-5202.

(c) Faculty of Education. University of Alicante.

doi:10.14198/jhse.2021.16.Proc3.55 


\section{INTRODUCTION}

Low back pain (LBP) is one of the most common musculoskeletal disorders, with a prevalence rate of $80 \%$ (Waddell G., 1987). The lifetime prevalence of LBP is high and more than $70 \%$ of adults have suffered LBP at some time in their lives (Dunn KM et al., 2013; Deyo RA et al., 1992; Buchbinder R et al., 2013). Low back pain is common worldwide, with $60-80 \%$ of people affected at some time in their lives (Beith ID et al., 2011; Lamb SE et al., 2010; Andersson GB et al., 1999). It has a major impact on health and health-related quality of life, diminishing the capacity for standing, walking and sitting (Gutke A et al., 2006; Scholich SL et al., 2012). In some patients, the initial acute pain may continue during a 3-month period and eventually develop into chronic LBP. Chronic LBP is associated with histomorphologic and structural changes in the paraspinalis muscles. Low back pain (LBP) is one of the most prevalent diseases afflicting people today. Moreover, the disabilities related to LBP are frequently associated with changes in the biomechanics of the lumbar spine. It has been shown that LBP patients reduce trunk rotation (van den Hoorn W et al., 2012; Muller R et al., 2015) and diminish the change in pelvis-thorax coordination from more in-phase to more anti-phase (Lamoth CJ et al., 2006) in the transverse plane, and spend more of the gait cycle in-phase coordination in the frontal plane (Seay JF et al., 2011) during level walking. Also, the range of motion (ROM) in the lumbar region has been found to have a reduction in LBP patients during stair climbing (Lee JK et al., 2011). These back muscles are smaller, contain fat, and show a degree of atrophic changes in select muscle fibres (Arokoski JP et al., 2001). Therefore, the lumbar paraspinalis muscles are weak with excessive fatigability (Roy SH et al., 1989; Lee HI et al., 2015). Furthermore, poor coordination of the para-spinalis muscles has been associated with chronic LBP (Magnusson ML et al., 1996). Exercise can improve back extension strength, mobility, endurance, and functional disability. (Manniche $C$ et al., 1988; Manniche $C$ et al., 1991). Exercise is used in chronic low back pain rehabilitation, in particular for training core stabilizing muscles including pelvic floor muscle (Hayden JA et al., 2005; Wang X et al., 2012; Hodges PW., 2003). Various exercises, such as lumbar stabilization exercise (SE), motor control exercise, core exercise, lumbar flexion exercise, walking exercise (WE), and bracing exercise, have been proposed to mitigate chronic LBP. These exercises focus on lumbar stabilization and core strengthening (O'Sullivan PB et al., 1997). Rehabilitative exercise focused on teaching and encouraging patients how to manage their LBP, and potentially prevent future recurrences, is frequently combined with SMT as an important aspect of promoting patient self-efficacy (Delitto A et al., 2012).

The purpose of this study was to evaluate and examine the effects of functional and traditional training on body composition and chronic low back pain treatment.

\section{METHODS}

A 33 old female subject presented complaining for severe chronic low back pain in the past 2 months. For several times she had to lie down on the floor because of low back pain crisis. Medical examination before exercise program including a spinal X-ray and MRI were conducted. Also, anthropometric and body composition evaluation using BIA (Tanita BC 601) were made before exercise intervention, 12, 24 and 36 weeks after exercise intervention.

\section{Exercise intervention program}

The total length of the program was 36 weeks with a training range session 3 days/week. Repetitions, sets, and rest periods were designed to provide a safe progression of resistance during the exercise intervention period. This program features alternating strength and endurance phases performed (progressing from $5-$ $20 \%$ of a patient's body weight). Total exercise training sessions were 45-60 min including warm-up (5-10 min cardio exercises/treadmill, elliptical step and bicycle) and cool down (5-10 min passive and active 
stretching). Trunk strength and endurance program were designed to load the trunk extensors in a graded manner. Also, the strength and endurance exercise program were goal-oriented, performance-based, and periodized. Table 1 shows some of the main exercise used to the intervention program. Exercises were individualised and have been applied as a combination of functional and traditional training.

Table 1. Functional and traditional training sample program.

\begin{tabular}{|c|c|c|c|}
\hline Exercise & 1-12-week program & 12-24-week program & 24-36-week program \\
\hline \multicolumn{4}{|l|}{ Functional exercises } \\
\hline \multicolumn{4}{|l|}{ Bosu ball plank } \\
\hline Bosu ball 3 points plank & $\begin{array}{c}1-4 \text { week } \\
(3 \times 30-45 \mathrm{sec})\end{array}$ & $\begin{array}{c}\text { 12-15 week } \\
(3 \times 60-75 \mathrm{sec})\end{array}$ & $\begin{array}{l}\text { 25-28 week } \\
(3 \times 75-90 \text { sec })\end{array}$ \\
\hline Side plank & $\begin{array}{c}5-8 \text { week } \\
(3 \times 45-60 \mathrm{sec})\end{array}$ & $\begin{array}{c}16-19 \text { week } \\
(3 \times 75-90 \mathrm{sec})\end{array}$ & $\begin{array}{c}16-19 \text { week } \\
(3 \times 90-115 \mathrm{sec})\end{array}$ \\
\hline Reverse salamander & $\begin{array}{c}\text { 9-12 week } \\
(3 \times 60-90 \mathrm{sec})\end{array}$ & $\begin{array}{c}20-24 \text { week } \\
(3 \times 90-115 \mathrm{sec})\end{array}$ & $\begin{array}{c}20-24 \text { week } \\
(3 \times 115-130 \mathrm{sec})\end{array}$ \\
\hline \multicolumn{4}{|l|}{ Swiss ball stir the pot } \\
\hline \multicolumn{4}{|l|}{ Functional exercises } \\
\hline Glute bridge & $\begin{array}{c}1-4 \text { week } \\
(3 \times 10-12 \text { reps } / 20-30 \mathrm{~kg})\end{array}$ & $\begin{array}{c}12-15 \text { week } \\
(3 \times 10-12 \text { reps } / 30-40 \mathrm{~kg})\end{array}$ & $\begin{array}{c}25-28 \text { week } \\
(3 \times 10-12 \text { reps } / 40-50 \mathrm{~kg})\end{array}$ \\
\hline Standing Russian twist & $\begin{array}{c}5-8 \text { week } \\
(3 \times 12-15 \text { reps } 5-10 \mathrm{~kg})\end{array}$ & $\begin{array}{c}16-19 \text { week } \\
(3 \times 12-15 \text { reps } 10-12.5)\end{array}$ & $\begin{array}{c}16-19 \text { week } \\
(3 \times 15-20 \text { reps } / 15 \mathrm{~kg})\end{array}$ \\
\hline Bosu ball mountain climber & $\begin{array}{c}\text { 9-12 week } \\
(4 \times 12-15 \text { reps })\end{array}$ & $\begin{array}{c}\text { 20-24 week } \\
(4 \times 15-20 \text { reps }) \\
\end{array}$ & $\begin{array}{c}\text { 20-24 week } \\
(4 \times 20-30 \text { reps })\end{array}$ \\
\hline \multicolumn{4}{|l|}{ Traditional exercises } \\
\hline Back extension & $\begin{array}{c}1-4 \text { week } \\
(3 \times 10-12 \text { reps } / 20 \mathrm{~kg})\end{array}$ & $\begin{array}{c}12-15 \text { week } \\
(3 \times 10-12 \text { reps } 25 \mathrm{~kg})\end{array}$ & $\begin{array}{c}25-28 \text { week } \\
(3 \times 10-12 \text { reps } / 30 \mathrm{~kg}\end{array}$ \\
\hline Lateral Pull-down & $\begin{array}{c}\text { 5-8 week } \\
(3 \times 12-15 \mathrm{reps} / 20 \mathrm{~kg})\end{array}$ & $\begin{array}{c}16-19 \text { week } \\
(3 \times 12-15 \mathrm{reps} / 25 \mathrm{~kg})\end{array}$ & $\begin{array}{c}16-19 \text { week } \\
(3 \times 12-15 \text { reps/30kg) }\end{array}$ \\
\hline Seated rowing machine & $\begin{array}{c}\text { 9-12 week } \\
(4 \times 12-15 \mathrm{reps} / 20 \mathrm{~kg})\end{array}$ & $\begin{array}{c}20-24 \text { week } \\
(4 \times 12-15 \text { reps } / 25 \mathrm{~kg})\end{array}$ & $\begin{array}{c}\text { 20-24 week } \\
(4 \times 12-15 \text { reps } 30 \mathrm{~kg})\end{array}$ \\
\hline Dumbbell bench press $\left(45^{\circ}\right)$ & $\begin{array}{c}1-4 \text { week } \\
(3 \times 8-10 \text { reps/4 kg) }\end{array}$ & $\begin{array}{c}12-15 \text { week } \\
(3 \times 10-12 \text { reps } / 4-6 \mathrm{~kg})\end{array}$ & $\begin{array}{c}25-28 \text { week } \\
(3 \times 10-12 \text { reps } / 8-10 \mathrm{~kg})\end{array}$ \\
\hline Dumbbell fly $\left(45^{\circ}\right)$ & $\begin{array}{c}\text { 5-8 week } \\
(3 \times 12-15 \text { reps } / 4 \mathrm{~kg})\end{array}$ & $\begin{array}{c}16-19 \text { week } \\
(3 \times 12-15 \text { reps/6-8 kg) }\end{array}$ & $\begin{array}{c}16-19 \text { week } \\
(3 \times 12-15 \text { reps } / 8-10 \mathrm{~kg})\end{array}$ \\
\hline Bench press & $\begin{array}{c}9-12 \text { week } \\
(4 \times 12-15 \text { reps/4-6) }\end{array}$ & $\begin{array}{c}20-24 \text { week } \\
(3 \times 12-15 \text { reps } / 6-8 \mathrm{~kg})\end{array}$ & $\begin{array}{c}\text { 20-24 week } \\
(3 \times 12-15 \mathrm{reps} / 8-10 \mathrm{~kg})\end{array}$ \\
\hline
\end{tabular}

\section{RESULTS}

Anthropometric and body composition evaluation results after 36 weeks exercise training show an improvement in:

- Weight from $109.1 \mathrm{~kg}$ to 90.8 after 36 weeks

- BMl from 36 to 29.47

- Total BF (\%) from 43.7 to 29

- Metabolic age from 47 to 40

- Visceral fat from 10 to $4(p<.05)$ (More details are shown in Table 2) 
Table 2. Anthropometric and body composition evaluation (Tanita BC 601).

\begin{tabular}{lcccc}
\hline & $\begin{array}{c}\text { Before } \\
\text { exercise } \\
\text { intervention }\end{array}$ & $\begin{array}{c}\text { 12 weeks } \\
\text { after exercise } \\
\text { intervention }\end{array}$ & $\begin{array}{c}\text { 24 weeks } \\
\text { after } \\
\text { intervention }\end{array}$ & $\begin{array}{c}\text { 36 weeks } \\
\text { after } \\
\text { intervention }\end{array}$ \\
\hline Weight (kg) & 109.1 & 106.1 & 97.7 & 90.8 \\
Height (m) & 1.77 & 1.77 & 1.77 & 1.77 \\
BMl & 36 & 34.35 & 31.85 & 29.47 \\
Waist circumference (cm) & 115 & 108 & 102 & 96.5 \\
Total BF (\%) & 43.7 & 38 & 33 & 29 \\
Muscle mass (kg) & 58.3 & 60 & 62 & 63.5 \\
Bone mass (kg) & 3.1 & 3.2 & 3.2 & 3.2 \\
Metabolic age (years) & 47 & 44 & 42 & 40 \\
Total body water (\%) & 42.7 & 45 & 46 & 48 \\
Visceral fat & 10 & 8 & 6 & 4 \\
Right arm (subcutaneous) fat (\%) & 48.2 & 44.8 & 42.9 & 39 \\
Right arm muscle mass (kg) & 3 & 3.1 & 3.2 & 3.2 \\
Left arm (subcutaneous) fat (\%) & 48.5 & 45 & 43 & 38 \\
Left arm muscle mass (kg) & 2.9 & 3 & 3.1 & 3.1 \\
Right leg (subcutaneous) fat (\%) & 45.6 & 42.7 & 39.6 & 37 \\
Right leg muscle mass $(\mathrm{kg})$ & 9.2 & 9.4 & 9.6 & 9.8 \\
Left leg (subcutaneous) fat (\%) & 45.6 & 42 & 38 & 36 \\
Left leg muscle mass (kg) & 9.4 & 9.5 & 9.7 & 9.9 \\
Total trunk fat (\%) & 45.6 & 41.3 & 38.1 & 34 \\
Total trunk muscle mass (kg) & 28.6 & 30 & 31 & 32 \\
\hline
\end{tabular}

Also, medical examination (spinal $\mathrm{X}$-ray and MRI) before exercise intervention program showed: $\mathrm{X}$-ray examination in Lumbo-sacral (X-ray, X-ray pedis), prescription: Noted a verticalization in the lumbar area; There were no visual bone structural changes in lumbar vertebras and both talocrural joints; Noted a reduction in the intravertebral area on L5-S1 region.

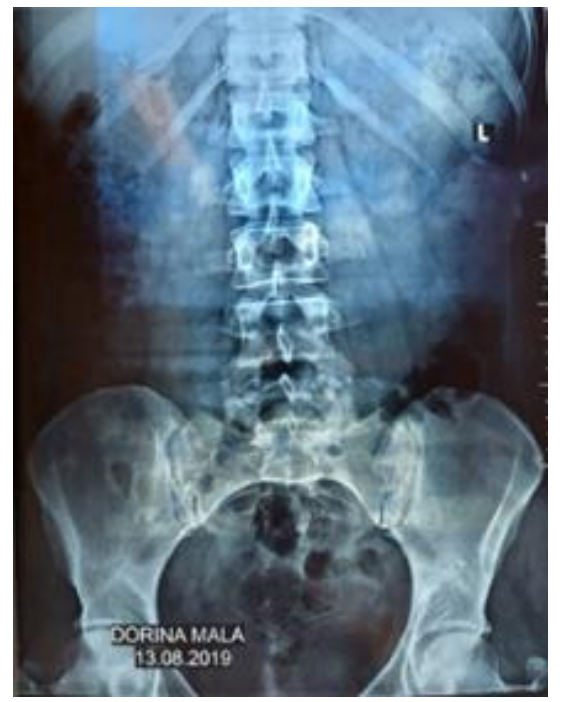

Figure 1. X-ray examination before intervention. 
MRI before exercise intervention results showed:

Modus: Examination conducted with sequences; $\mathrm{T} 1$ tse, $\mathrm{T} 2$ tse, T2 tirm with axial cut, concluded: A reduction in the physiological lumbar lordosis; Normal vertebral anatomy and inclination; Neural posterior arch integrity is normal; Cortexes and end line bords without changes; Discal prolabition foraminal dexter in L4/L5; Posterior discal prolabition in L5/S1; Bone marrow with normal signal; No lesions in the pre-vertebral soft tissues.

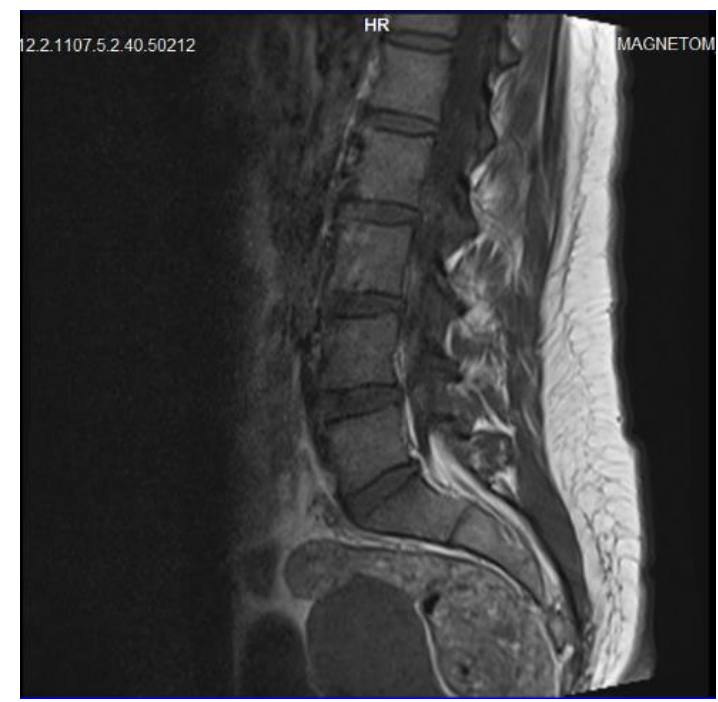

Figure 2. MRI before exercise intervention.

Spinal X-ray 36 weeks after intervention results showed no changes in: Thoracic- lumbar column in axis; Verticalization in the lumbar area; Vertebral bodies with preserved normal height; Reduction in the intravertebral area on L5-S1 region.

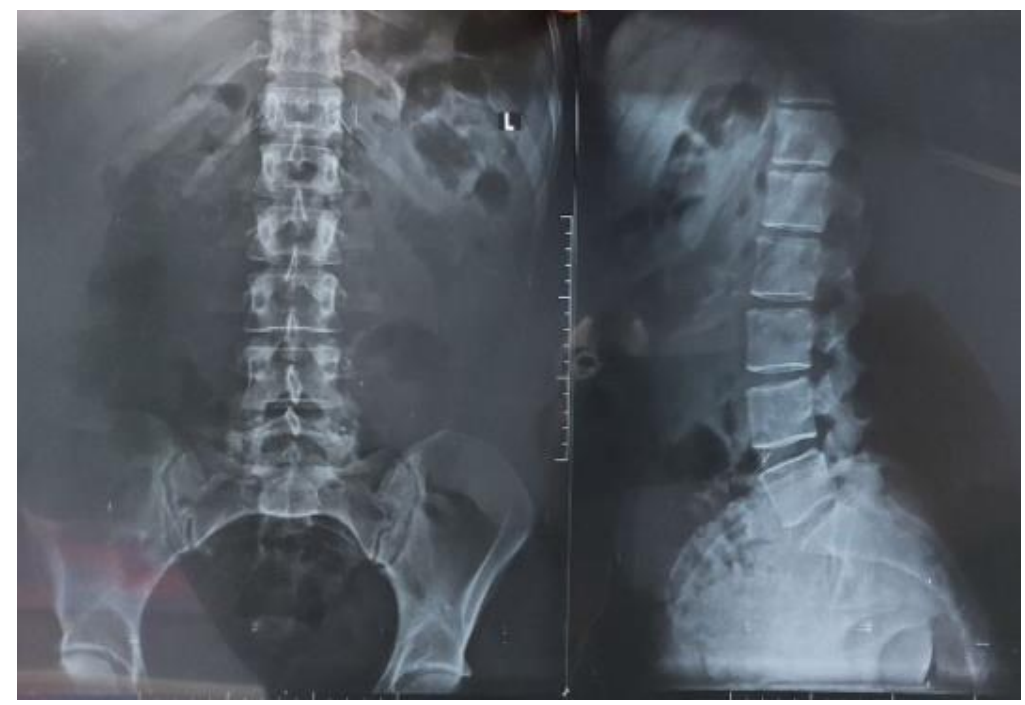

Figure 3. X-ray examination after intervention.

Also, MRI after 36 weeks after exercise intervention results showed no changes in: A reduction in the physiological lumbar lordosis; Normal vertebral anatomy and inclination; Neural posterior arch integrity is 
normal; Cortexes and end line bords without changes; Discal prolabition foraminal dexter in L4/L5; Posterior discal prolabition in L5/S1; Bone marrow with normal signal; No lesions in the pre-vertebral soft tissues.

Figure 4: MRI after exercise intervention.

\section{DISCUSSION AND CONCLUSION}

Based on the data result, after 3 weeks total body composition, decreasing total body weight and increasing total muscle mass and subjects was not complaining for low back pain crisis. X-ray and MRI after 36 weeks after exercise intervention results showed no changes but the exercise intervention program helped her to improve her daily life and be more focused and productive in her work task which involves more weight carrying, longer work hours. She is still practicing and enjoying the health benefits of exercise training. Based on the data results we can say that exercise functional and traditional training program had a positive effect on body composition and chronic low back pain treatment. It is very important that this exercise training programs should be prescribed from appropriate and licensed rehabilitation specialist and should be personalized for the specific individual needs in order to achieve the best and necessary results. Results of this case study suggest that further studies using functional training intervention program with a larger sample should be conducted.

\section{REFERENCES}

Andersson GB. Epidemiological features of chronic low-back pain. Lancet 1999; 354: 581-585. https://doi.org/10.1016/S0140-6736(99)01312-4

Arokoski JP, Valta T, Airaksinen O, et al. Back and abdominal musclefunction during stabilization exercises. Arch Phys Med Rehabil 2001; 82: 1089-98. https://doi.org/10.1053/apmr.2001.23819

Beith ID, Kemp A, Kenyon J, et al. Identifying neuropathic back and leg pain: a cross sectional study. Pain 2011; 152: 1511-1516. https://doi.org/10.1016/j.pain.2011.02.033

Buchbinder R, Blyth FM, March LM et al: Placing the global burden of low back pain in context. Best Pract Res Clin Rheumatol, 2013; 27(5): 575-89. https://doi.org/10.1016/j.berh.2013.10.007

Delitto A, George SZ, Van Dillen LR, Whitman JM, Sowa G, Shekelle P, Denninger TR, Godges JJ. Low back pain. J Orthop Sports Phys Ther 2012;42(4):A1-57. https://doi.org/10.2519/jospt.2012.42.4.A1

Deyo RA, Rainville J, Kent DL: What can the history and physical examination tell us about low back pain. JAMA, 1992; 268(6): 760-65. https://doi.org/10.1001/jama.268.6.760

Dunn KM, Hestbaek L, Cassidy JD: Low back pain across the life course. Best Pract Res Clin Rheumatol, 2013; 27(5): 591-600. https://doi.org/10.1016/j.berh.2013.09.007

Gutke A, Ostgaard HC and Oberg B. Pelvic girdle pain and lumbar pain in pregnancy: a cohort study of the consequences in terms of health and functioning. Spine (Phila Pa 1976) 2006; 31: E149-E155. https://doi.org/10.1097/01.brs.0000201259.63363.e1

Hayden JA, van Tulder MW, Malmivaara A, et al. Exercise therapy for treatment of nonspecific low back pain. Cochrane Database Syst Rev 2005; 3: CD000335. https://doi.org/10.1002/14651858.CD000335.pub2

Hodges PW. Core stability exercise in chronic low back pain. Orthop Clin North Am 2003; 34: 245-254. https://doi.org/10.1016/S0030-5898(03)00003-8

Lamb SE, Hansen Z, Lall R, et al. Group cognitive behavioural treatment for low-back pain in primary care: a randomised controlled trial and cost-effectiveness analysis. Lancet 2010; 375: 916-923. https://doi.org/10.1016/S0140-6736(09)62164-4 
Lamoth CJ, Meijer OG, Daffertshofer A et al: Effects of chronic low back pain on trunk coordination and back muscle activity during walking: Changes in motor control. Eur Spine J, 2006; 15(1): 23-40 https://doi.org/10.1007/s00586-004-0825-y

Lee HI, Lee ST, Kim M, et al. Sex differences in predicting chronicity oflow-back pain after acute trauma using lumbar muscle area. Am J PhysMed Rehabil 2015;94:123-30. https://doi.org/10.1097/PHM.0000000000000149

Lee JK, Desmoulin GT, Khan AH, Park EJ: Comparison of 3D spinal motions during stair-climbing between individuals with and without low back pain. Gait Posture, 2011; 34(2): 222-26. https://doi.org/10.1016/i.gaitpost.2011.05.002

Magnusson ML, Aleksiev A, Wilder DG, et al. European Spine Society-the AcroMed Prize for Spinal Research 1995. Unexpected load andasymmetric posture as etiologic factors in low back pain. Eur Spine J1996;5:23-35. https://doi.org/10.1007/BF00307824

Manniche C, Hesselsoe G, Bentzen L, et al. Clinical trial of intensive muscle training for chronic low back pain. Lancet 1988;2:1473-6. https://doi.org/10.1016/S0140-6736(88)90944-0

Manniche C, Lundberg E, Christensen I, et al. Intensive dynamic back exercises for chronic low back pain: a clinical trial. Pain 1991;47:53-63. https://doi.org/10.1016/0304-3959(91)90011-L

Muller R, Ertelt T, Blickhan R: Low back pain affects trunk as well as lower limb movements during walking and running. J Biomech, 2015; 48(6): 1009-14. https://doi.org/10.1016/j.jbiomech.2015.01.042

O'Sullivan PB, Phyty GD, Twomey LT, et al. Evaluation of specific stabilizing exercise in the treatment of chronic low back pain with radiologic diagnosis of spondylolysis or spondylolisthesis. Spine (PhilaPa 1976) 1997; 22:2959-67. https://doi.org/10.1097/00007632-199712150-00020

Roy SH, De Luca CJ, Casavant DA. Lumbar muscle fatigue and chroniclower back pain. Spine (Phila Pa 1976) 1989;14:992-1001. https://doi.org/10.1097/00007632-198909000-00014

Scholich SL, Hallner D, Wittenberg RH, et al. The relationship between pain, disability, quality of life and cognitive-behavioural factors in chronic back pain. Disabil Rehabil 2012; 34: 1993-2000. https://doi.org/10.3109/09638288.2012.667187

Seay JF, Van Emmerik RE, Hamill J: Influence of low back pain status on pelvis-trunk coordination during walking and running. Spine, 2011; 36(16): E1070-79. https://doi.org/10.1097/BRS.0b013e3182015f7C

van den Hoorn W, Bruijn SM, Meijer OG et al: Mechanical coupling between transverse plane pelvis and thorax rotations during gait is higher in people with low back pain. J Biomech, 2012; 45(2): 342-47. https://doi.org/10.1016/i.jbiomech.2011.10.024

Wang X, Zheng J, Liu J, et al. Effect of core stability training on patients with chronic low back pain. HealthMED 2012; 6: 754-759.

\section{(2) $(0 \Theta \Theta$}

This work is licensed under a Attribution-NonCommercial-NoDerivatives 4.0 International (CC BY-NC-ND 4.0). 